\title{
miR-671-3p is downregulated in non-small cell lung cancer and inhibits cancer progression by directly targeting CCND2
}

\author{
YUANSHAN YAO $^{1}$, YINJIE ZHOU $^{1}$ and XIAOJUN FU ${ }^{2}$ \\ Departments of ${ }^{1}$ Thoracic Surgery and ${ }^{2}$ Gastrointestinal Surgery, Ningbo No. 2 Hospital, \\ Ningbo, Zhejiang 300270, P.R. China
}

Received May 22, 2018; Accepted December 10, 2018

DOI: $10.3892 / \mathrm{mmr} .2019 .9858$

\begin{abstract}
MicroRNAs (miRNAs) are implicated in the development and progression of non-small cell lung cancer (NSCLC). A previous study suggested that miR-671-3p suppresses the development of breast cancer. However, the role of miR-671-3p in NSCLC remains largely unknown. In the present study, it was identified that miR-671-3p was significantly upregulated in NSCLC tissues compared with adjacent normal tissues by reverse transcription quantitative polymerase chain reaction (RT-qPCR). Similarly, decreased levels of miR-671-3p in NSCLC cell lines were observed compared with those in the non-tumorigenic human bronchial epithelial NL20 cell line. Cell Counting Kit- 8 and Transwell invasion assays indicated that miR-671-3p overexpression suppressed the proliferation and invasion of A549 cells, and vice versa. Mechanistically, it was demonstrated that CCND2 was a direct target of miR-671-3p. RT-qPCR and western blot analysis indicated that miR-671-3p overexpression decreased the expression of CCND2 in A549 cells. Furthermore, rescue experiments demonstrated that the restoration of CCND2 may significantly reverse the suppressive roles of miR-671-3p overexpression on NSCLC cell proliferation and invasion. Taken together, the present study demonstrated that miR-671-3p exerted its tumor-suppressive roles via directly targeting CCND2 in NSCLC.
\end{abstract}

\section{Introduction}

Lung cancer is one of the most prevalent and aggressive types of cancer worldwide, with an extremely high mortality rate (1). Regardless of the advances in surgical techniques and chemotherapy, the outcomes of patients with lung cancer remain poor, and the 5 -year survival rate is only $15 \%$ (2). Metastasis is the most important risk for cancer-associated mortality in

Correspondence to: Dr Xiaojun Fu, Department of Gastrointestinal Surgery, Ningbo No. 2 Hospital, 41 Northwest Street, Ningbo, Zhejiang 300270, P.R. China

E-mail: fuxiaojun6565@163.com

Key words: miR-671-3p, non-small cell lung cancer, proliferation, invasion, CCND2 lung cancer (3). Among all cases, non-small cell lung cancer (NSCLC) accounts for almost 85\% (4). Therefore, to improve the therapy available to patients, exploration of the molecular mechanisms underlying NSCLC development and progression is urgently required.

MicroRNAs (miRNAs) are a type of short noncoding RNAs measuring 19-22 nucleotides long, which function by recognizing specific complementary sites in the 3'-untranslated region (3'-UTR) of target mRNAs (5). Through directing the degradation of target mRNAs, miRNAs are involved in the regulation of gene expression. Accumulating evidence has indicated that miRNAs serve crucial functions in multiple biological processes, including proliferation, migration, development and survival (6). In previous decades, miRNAs have been demonstrated to participate in tumorigenesis and an increasing number of previous studies have suggested that dysregulation of miRNAs is associated with the pathogenesis of cancer (7). Furthermore, a previous study has indicated that miRNAs exert vital roles in NSCLC occurrence and progression (8). For example, miR-520a-3p inhibits cell growth and metastasis of non-small cell lung cancer through the phosphoinositide 3'-kinase/protein kinase B (AKT)/mechanistic target of rapamycin signaling pathway (9). miR-224 enhances invasion and metastasis by targeting homeobox D10 in non-small cell lung cancer cells (10). Therefore, the identification of important miRNAs in NSCLC will contribute to understanding of the pathogenesis and novel drug identification.

A recent study indicated that miR-671-3p inhibits the development of breast cancer (11). The role of miR-671-3p in NSCLC remains largely unknown. The present study aimed to investigate the biological function of miR-671-3p in NSCLC progression. It was identified that miR-671-3p was significantly upregulated in NSCLC tissues compared with adjacent normal tissues. Furthermore, it was demonstrated that miR-671-3p inhibited NSCLC cell proliferation and invasion via directly targeting cyclin D2 (CCND2). In conclusion, the present study demonstrated that miR-671-3p exerts tumor-suppressive roles in NSCLC, suggesting miR-671-3p may be a promising therapeutic target.

\section{Materials and methods}

Patient samples. A total of 43 NSCLC cancer and corresponding adjacent tissue specimens were obtained from 
patients with NSCLC who underwent curative resection at Ningbo No. 2 Hospital (Ningbo, China). No patients had undergone chemotherapy or radiotherapy prior to surgery. Fresh samples were collected at the time of surgery, and were rapidly frozen in liquid nitrogen and stored at $-80^{\circ} \mathrm{C}$ until use. The clinicopathological characteristics of the patients with NSCLC are summarized in Table I. Informed consent was signed by each patient. The present study was approved by the Ethics Committee of Ningbo No. 2 Hospital.

Cell culture. Human NSCLC A549, H1299, H1650 and H1975 cell lines and the non-tumorigenic human bronchial epithelial NL20 cell line were purchased from the American Type Culture Collection (Manassas, VA, USA). All the cells were cultured in RPMI-1640 (Gibco; Thermo Fisher Scientific, Inc., Waltham, MA, USA) supplemented with penicillin (100 U/ml), streptomycin (100 U/ml) (Sigma-Aldrich; Merck KGaA, Darmstadt, Germany), and $10 \%$ fetal bovine serum (Gibco; Thermo Fisher Scientific, Inc.) at $37^{\circ} \mathrm{C}$ in $5 \% \mathrm{CO}_{2}$.

miRNA transfection. miR-671-3p mimics, miR-671-3p negative control (miR-NC), miR-671-3p inhibitors and miR-671-3p inhibitor negative control (anti-miR-NC) were purchased from Guangzhou RiboBio Co., Ltd. (Guangzhou, China). These were individually and transiently transfected with A549 cells at a final concentration of $100 \mathrm{nM}$ using Lipofectamine ${ }^{\circledR} 2000$ reagent (Invitrogen; Thermo Fisher Scientific, Inc.). The miR-671-3p inhibitors were modified antisense oligonucleotides designed specifically to bind to and inhibit endogenous miR-671-3p with a rare off-target effect: miR-NC: 5'-ACAUCUGCGUAAGAU UCGAGUCUA-3'; miR-671-3p mimics: 5'-UCCGGUUCU CAGGGCUCCACC-3'; anti-miR-NC: 5'-GCGTAACTAATA CATCGGATTCGT-3'; miR-671-3p inhibitors: 5'-GGUGGA GCCCUGAGAACCGGA-3'. The efficiency was measured $48 \mathrm{~h}$ after transfection, by reverse transcription-quantitative polymerase chain reaction (RT-qPCR) as described below.

$R T-q P C R$. Total RNA was isolated from tissues or cultured cells using TRIzol ${ }^{\circledR}$ reagent (Thermo Fisher Scientific, Inc.). RNA quality and concentration was measured using a Nanodrop 2000 spectrophotometer (Thermo Fisher Scientific, Inc., Wilmington, DE,USA). A total of $1 \mu \mathrm{g}$ RNA was used for cDNA synthesis using a PrimeScript 1st Strand cDNA Synthesis kit (Takara Bio, Inc., Otsu, Japan). qPCR was performed using the miRNA Q-PCR Detection kit (GeneCopoeia, Inc., Rockville, MD, USA). The PCR thermocycling conditions were: $95^{\circ} \mathrm{C}$ for $30 \mathrm{sec}$, followed by 40 cycles of $95^{\circ} \mathrm{C}$ for $5 \mathrm{sec}$ and $60^{\circ} \mathrm{C}$ for $34 \mathrm{sec}$. The RT-qPCR data were analyzed using the $2^{-\triangle \triangle \mathrm{Cq}}$ method (12) and relative to the small nuclear RNA U6 or GAPDH levels. RT primers were as follows: GAPDH forward (F), 5'-ACAACTTTGGTATCG TGGAAGG-3'; GAPDH reverse (R), 5'-GCCATCACGCCA CAGTTTC-3'; U6 F, 5'-CTCGCTTCGGCAGCACA-3'; U6 R, 5'-AACGCTTCACGAATTTGCGT-3'; miR-671-3p F, 5'-CTG GCTGGACAGAGTTGTCAT-3'; miR-671-3p R, 5'-TCCGGT TCTCAGGGCTCCACC-3'; CCND2 F, 5'-TACCTGGACCGT TTCTTGGC-3'; CCND2 R, 5'-AGGCTTGATGGAGTTGTC GG-3'.

Western blot analysis. Western blot was performed as previously described (13). In brief, tumor cells were lysed with lysis buffer
(0.5 mol/1 Tris- $\mathrm{HCl}, \mathrm{pH} 7.4,1.5 \mathrm{~mol} / \mathrm{l} \mathrm{NaCl}, 2.5 \%$ deoxycholic acid, 10\% NP-40 and $10 \mathrm{mmol} / \mathrm{l}$ EDTA) in the presence of cocktail protease inhibitors (Thermo Fisher Scientific, Inc.). The lysates were collected by centrifugation at $16,000 \mathrm{x}$ g for $20 \mathrm{~min}$ at $4^{\circ} \mathrm{C}$. Protein concentration was determined by Bio-Rad Protein Assay kit (Bio-Rad Laboratories, Inc., Hercules, CA, USA). A total of $50 \mathrm{mg}$ protein/lane was separated by $12 \%$ SDS-PAGE and transferred to a polyvinylidene difluoride membrane (Thermo Fisher Scientific, Inc.) The membrane was blocked with 5\% non-fat dry milk (Yili Group, Beijing, China) for $1 \mathrm{~h}$ at room temperature, followed by incubation with primary antibodies for $2 \mathrm{~h}$ at room temperature: Anti-Cyclin D2 (1:1,000; cat. no. ab207604; Abcam, Cambridge, MA, USA) and anti-GAPDH (1:1,000; cat. no. ab9485; Abcam). Following washing, the membrane was incubated with horseradish peroxidase-conjugated goat anti-rabbit secondary antibody (1:5,000; cat. no. ab7090; Abcam) at room temperature for $1 \mathrm{~h}$. An enhanced chemiluminescence kit (Thermo Fisher Scientific, Inc.) was used to perform chemiluminescence detection.

Cell Counting Kit-8 (CCK-8) proliferation assays. Each group of A549 cells was collected at 24, 48, 72 and $96 \mathrm{~h}$ following transfection. Then, cells were incubated with $10 \mu \mathrm{l} \mathrm{CCK}-8$ reagent (Beyotime Institute of Biotechnology, Haimen, China) for $2 \mathrm{~h}$ at $37^{\circ} \mathrm{C}$. Next, absorbance at $450 \mathrm{~nm}$ was measured at each time point using an enzyme immunoassay analyzer. The experiment was conducted in three separate wells for each sample, and performed in triplicate.

Transwell invasion assays. To measure cell invasion, a Transwell invasion chamber coated with Matrigel ${ }^{\circledR}$ (Corning Incorporated, Corning, NY, USA) at $37^{\circ} \mathrm{C}$ for $30 \mathrm{~min}$ was used to determine the cell invasion ability. Following fixation with $4 \%$ paraformaldehyde for $1 \mathrm{~h}$ at room temperature, the cells that had invaded the membrane were stained with $0.1 \%$ crystal violet for $30 \mathrm{~min}$ at room temperature and counted. The number of cells that had invaded through the Matrigel was counted in 5 fields of triplicate membranes at magnification, x100, using an inverted light microscope (Olympus Corporation, Tokyo, Japan).

Luciferase assay. The potential targets and binding sites of miR-671-3p were analyzed using TargetScan7 tool (http://www.targetscan.org/vert_71/). The 3'-UTR of the CCND2 was obtained by gene synthesis, and inserted downstream of the luciferase reporter gene in a pmirGLO vector (Promega Corporation, Madison, WI, USA). For the luciferase reporter assay, A549 cells $\left(2 \times 10^{4} /\right.$ well) were seeded in a 24-well plate and incubated for $24 \mathrm{~h}$ prior to transfection. Next, firefly luciferase constructs containing the 3'-UTR and miR-671-3p mimics or the corresponding negative controls were co-transfected into A549 cells using Lipofectamine ${ }^{\circledR}$ 2000 (Invitrogen; Thermo Fisher Scientific, Inc.). Cells were collected at $48 \mathrm{~h}$ after transfection, and measured using the Dual-Luciferase Reporter System (Promega Corporation), according to manufacturer's protocols. The pRL-TK Renilla luciferase activity was used for normalization.

Statistical analysis. All statistical analyses were performed using SPSS 20.0 (IBM Corp., Armonk, NY, USA) and 
Table I. Associations between miR-671-3p expression and clinicopathological characteristics of patients with non-small cell lung cancer.

\begin{tabular}{|c|c|c|c|}
\hline \multirow[b]{2}{*}{$\begin{array}{l}\text { Clinicopathological } \\
\text { features }\end{array}$} & \multicolumn{2}{|c|}{$\begin{array}{l}\text { miR-671-3p } \\
\text { expression }\end{array}$} & \multirow[b]{2}{*}{ P-value } \\
\hline & $\begin{array}{l}\text { Lower } \\
(n=21)\end{array}$ & $\begin{array}{l}\text { Higher } \\
(n=22)\end{array}$ & \\
\hline Sex & & & 0.719 \\
\hline Male & 15 & 17 & \\
\hline Female & 6 & 4 & \\
\hline Age, years & & & 0.373 \\
\hline$\leq 60$ & 14 & 12 & \\
\hline$>60$ & 7 & 11 & \\
\hline Lymph node metastasis & & & 0.015 \\
\hline Negative & 6 & 15 & \\
\hline Positive & 15 & 7 & \\
\hline Tumor size, $\mathrm{cm}$ & & & 0.033 \\
\hline$\leq 3$ & 8 & 16 & \\
\hline$>3$ & 13 & 6 & \\
\hline $\begin{array}{l}\text { Tumor node } \\
\text { metastasis stage }\end{array}$ & & & 0.034 \\
\hline $\mathrm{I} / \mathrm{II}$ & 7 & 15 & \\
\hline III & 14 & 7 & \\
\hline
\end{tabular}

The median value of miR-671-3p level was chosen as the cut-off value. P-values were obtained using $\chi^{2}$ test. miR, microRNA.

GraphPad Prism v. 6 (GraphPad Software, Inc., La Jolla, CA, USA). A Student's t-test and one-way analysis of variance followed by Tukey's post-hoc test were used to analyze two or multiple groups, respectively. Kaplan-Meier curves were used to analyze survival rate and log-rank tests was used to calculate the corresponding P-values. Associations between miR-671-3p expression and clinicopathological characteristics of patients with NSCLC were analyzed using a $\chi^{2}$ test. $\mathrm{P}<0.05$ was considered to indicate a statistically significant difference.

\section{Results}

miR-671-3p is downregulated in NSCLC tissues. In order to explore the function of miR-671-3p in NSCLC progression, the expression of miR-671-3p in 43 pairs of NSCLC tissues and adjacent normal tissues was first analyzed by RT-qPCR. The results demonstrated that miR-671-3p expression was significantly downregulated in NSCLC tissues compared with the adjacent normal tissues (Fig. 1A). Consistently, it was identified that the expression of miR-671-3p was also markedly downregulated in the NSCLC A549, H1975, H1299 and H1650 cell lines compared with the non-tumorigenic human bronchial epithelial NL20 cell line (Fig. 1B). Furthermore, the NSCLC tissues were divided into miR-671-3p high and miR-671-3p low groups using the median value of miR-671-3p level as the cut-off value. pTNM staging designations were made according to the postsurgical pathological staging system according to the 7th edition of the TNM classification of malignant tumors (14). It was observed that the level of miR-671-3p was negatively associated with tumor size, Tumor Node Metastasis stage and metastasis (Table I). Taken together, these results suggested that miR-671-3p may be involved in NSCLC progression.

miR-671-3p overexpression inhibits NSCLC cell proliferation and invasion. To additionally investigate the biological functions of miR-671-3p, miR-671-3p was overexpressed in A549 cells, which exhibited the lowest level of miR-671-3p among all measured cell lines. RT-qPCR analysis indicated that miR-671-3p levels were significantly upregulated following transfection with miR-671-3p mimics in A549 cells (Fig. 2A). Then, CCK-8 and Transwell invasion assays were performed. The results indicated that ectopic expression of miR-671-3p significantly inhibited the proliferation and decreased the invading cell number (Fig. 2B and C), suggesting that miR-671-3p exerts a tumor-suppressive role in NSCLC.

miR-671-3p inhibition promotes NSCLC cell proliferation and invasion. To additionally confirm the role of miR-671-3p in NSCLC, experiments using miR-671-3p inhibitors were performed. As demonstrated by the RT-qPCR assay results, miR-671-3p expression was significantly downregulated in A549 cells following transfection with miR-671-3p inhibitors compared with the negative control (Fig. 3A). The CCK-8 assay revealed that miR-671-3p inhibition led to a decreased proliferation ability in A549 cells (Fig. 3B). Furthermore, knockdown of miR-671-3p significantly inhibited the invasion of A549 cells (Fig. 3C).

CCND2 is a target of miR-671-3p. The present study then aimed to determine the mechanism of miR-671-3p in NSCLC. The potential target of miR-671-3p was identified using TargetScan7. The results implied that CCND2 may be a target of miR-671-3p, as a potential binding site of miR-671-3p in the CCND2 3'-UTR was identified (Fig. 4A). To validate this prediction, a luciferase reporter assay was then performed. It was identified that the overexpression of miR-671-3p significantly repressed the luciferase intensity of CCND2-3'-UTR-wild type in A549 cells (Fig. 4B). Notably, mutation of the predicted site in CCND2 3'-UTR abrogated the effect of miR-671-3p (Fig. 4B), indicating that miR-671-3p interacts with CCND2 mRNA directly. In addition, RT-qPCR and western blot analyses suggested that the overexpression of miR-671-3p markedly decreased the mRNA and protein levels of CCND2 in A549 cells (Fig. 4C and D). The expression levels of CCND2 were also upregulated in NSCLC tissues and cell lines (Fig. 4E and F). Taken together, these results suggested that CCND2 was directly targeted by miR-671-3p in NSCLC cells.

$C C N D 2$ restoration rescued the effects of miR-671-3p overexpression. To confirm the role of CCND2 in the process of miR-671-3p-mediated NSCLC progression, rescue experiments were performed. The efficiency of CCND2 overexpression was first validated by RT-qPCR. The results revealed that the CCND2 mRNA level was significantly upregulated in A549 cells following transfection with the pCDNA3-CCND2 

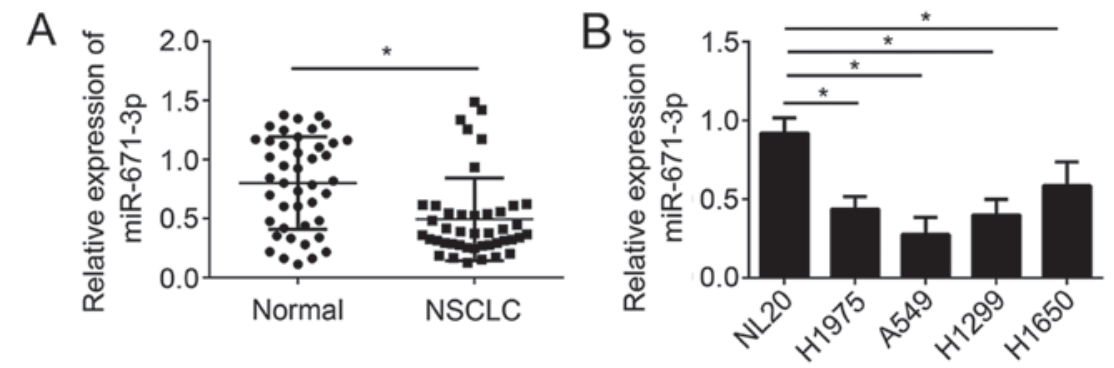

Figure 1. miR-671-3p is downregulated in NSCLC tissues. (A) Expression levels of miR-671-3p were measured in 43 pairs of NSCLC tissues and adjacent normal tissues using reverse transcription quantitative polymerase chain reaction analysis. (B) miR-671-3p expression was downregulated in NSCLC cell lines compared with NL20 cells. "P<0.05 vs. NL20. miR, microRNA; NSCLC, non-small cell lung cancer.

A

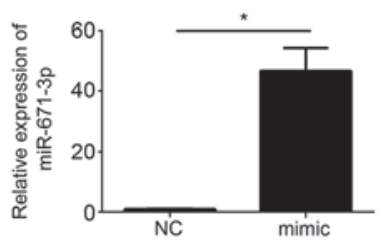

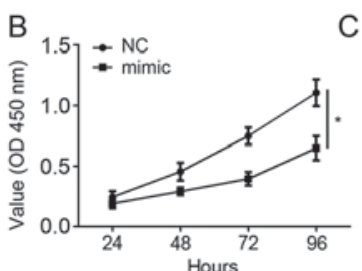
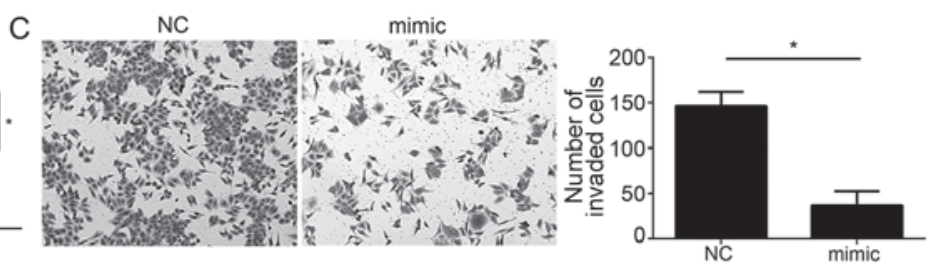

Figure 2. miR-671-3p overexpression inhibits non-small cell lung cancer cell proliferation and invasion. (A) Reverse transcription quantitative polymerase chain reaction analysis demonstrated that miR-671-3p expression was significantly upregulated following transfection with miR-671-3p mimics in A549 cells. (B) Cell Counting Kit-8 assay indicated that miR-671-3p overexpression inhibited A549 cell proliferation. (C) Transwell invasion assay demonstrated that transfection with miR-671-3p mimics led to decreased levels of cell invasion (magnification, $\mathrm{x} 100$ ). ${ }^{\text {"P }}<0.05$ vs. NC group. miR, microRNA; NC, negative control; OD, optical density.
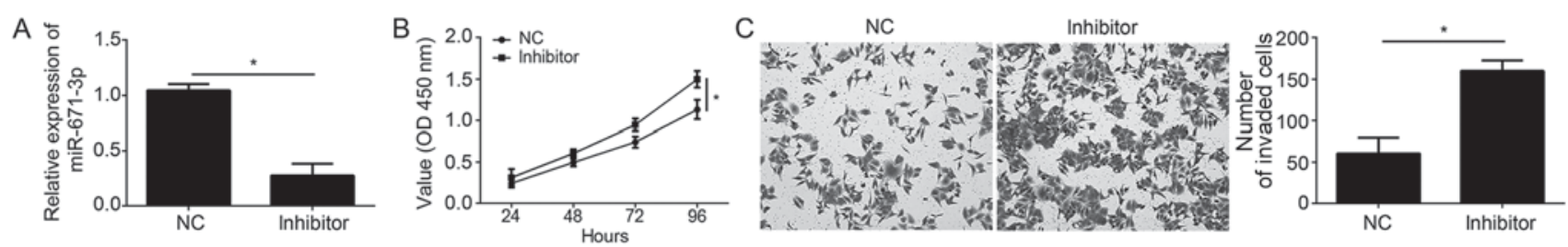

Figure 3. miR-671-3p inhibition promotes NSCLC cell proliferation and invasion. (A) Reverse transcription quantitative polymerase chain reaction analysis indicated that miR-671-3p expression was significantly downregulated following transfection with miR-671-3p inhibitors in A549 cells. (B) Ectopic expression of miR-671-3p inhibitors inhibited A549 cell proliferation. (C) Transwell invasion assay indicated that miR-671-3p knockdown suppressed A549 cell invasion

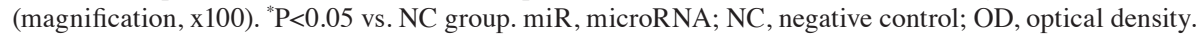

vector (Fig. 5A). Then, CCK-8 and Transwell invasion assays were conducted. The results demonstrated that miR-671-3p overexpression significantly inhibited the proliferation and invasion of NSCLC cells (Fig. 5B and C). However, restoration of CCND2 markedly rescued the inhibitory effects of miR-671-3p overexpression in A549 cells (Fig. 5B and C). In conclusion, these results demonstrated that miR-671-3p exerts its roles via directly targeting CCND2 in NSCLC cells.

\section{Discussion}

Lung cancer has become a major public health challenge due to its high incidence and mortality (15). Among all lung cancer cases, NSCLC accounts for $\sim 85 \%$ (1). Nevertheless, the molecular mechanism underlying lung cancer progression remains poorly understood. In previous decades, with advances in surgical techniques and the development of chemical drug therapies, the outcomes of NSCLC patients have been improved (16). However, the 5-year survival rate of patients with NSCLC is only $15 \%$, even following treatment (17). Therefore, determination of the molecular mechanism and development of novel therapeutic strategies are urgently required. In the present study, the association between miR-671-3p expression and NSCLC progression was demonstrated. It was identified that miR-671-3p expression was significantly downregulated in NSCLC tissues compared with adjacent normal tissues. Overexpression of miR-671-3p significantly suppressed the proliferation and invasion of NSCLC cells, and vice versa. These data suggested that miR-671-3p serves as a tumor suppressor, and implies that miR-671-3p may be a potential target for NSCLC treatment.

An increasing number of miRNAs have been recognized as oncogenes or tumor suppressors, which suggests that miRNAs may be promising targets for cancer intervention (18). For example, miR-30a was significantly downregulated in osteosarcoma, and suppressed osteosarcoma proliferation and metastasis by targeting myocyte enhancer factor 2D (19). miR-12528 was demonstrated to regulate tumorigenesis and metastasis in lung cancer by targeting insulin-like growth factor 1 receptor (20). miR-502 mediates esophageal cancer 
A

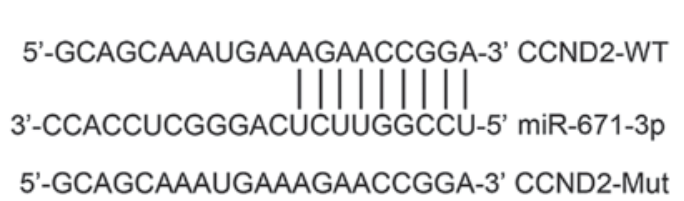

C

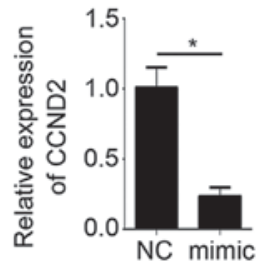

D

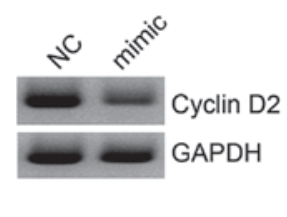

E

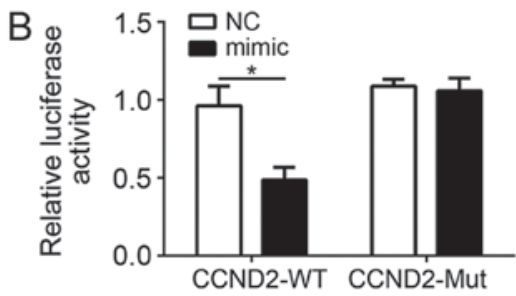

$\mathrm{F}$

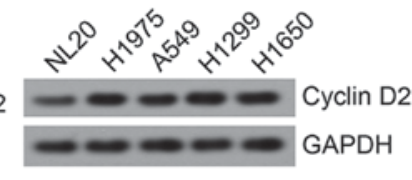

Figure 4. CCND2 is a target of miR-671-3p. (A) Predicted binding site of miR-671-3p in the 3'-untranslated region of CCND2. (B) Luciferase reporter assay indicated that miR-671-3p overexpression repressed the luciferase intensity of CCND2-WT reporter in A549 cells. (C) Reverse transcription quantitative polymerase chain reaction analysis indicated that miR-671-3p overexpression inhibited the mRNA level of CCND2 in A549 cells. (D) Western blot analysis suggested that miR-671-3p overexpression decreased the protein level of CCND2 in A549 cells. Western blot analysis indicated that CCND2 was upregulated in (E) NSCLC tissues and (F) cell lines. All experiments were repeated in triplicate. ${ }^{*} \mathrm{P}<0.05$ vs. NC group. miR, microRNA; NC, negative control; CCND2, cyclin D2; WT, wild type; Mut, mutant.

A

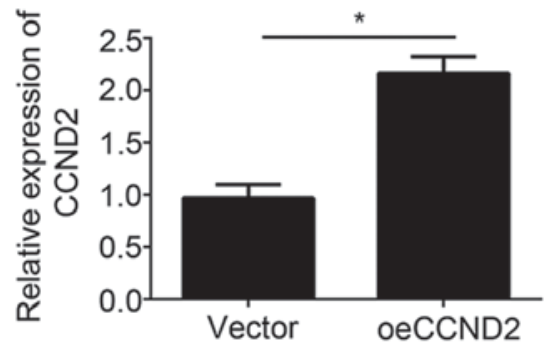

$\mathrm{B}$

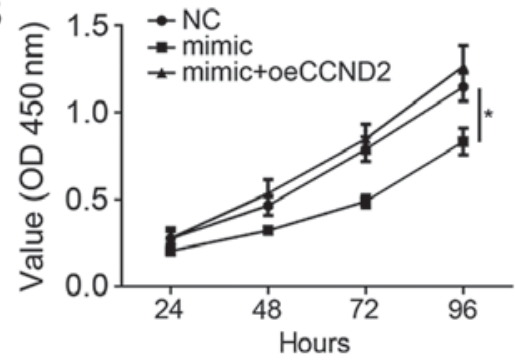

C

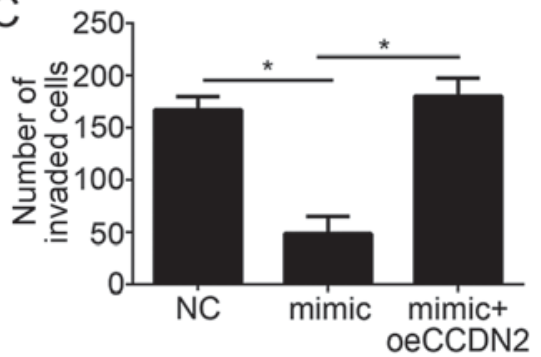

Figure 5. CCND2 restoration rescues the effects of miR-671-3p overexpression. (A) CCND2 expression was significantly upregulated following transfection with pCNDA3-CCND2 vector in A549 cells. (B) CCK-8 assays indicated that the restoration of CCND2 rescued the proliferation ability of A549 cells transfected with miR-671-3p mimics. (C) Transwell assay indicated that the ectopic expression of CCND2 increased the invasive potential of A549 cells transfected with miR-671-3p mimics. "P<0.05 vs. NC group. miR, microRNA; NC, negative control; CCND2, cyclin D2; oe, overexpressed; OD, optical density.

TE1 cell proliferation by promoting AKT phosphorylation (21). Therefore, it is crucial to investigate the association between miRNA and cancer. A previous study indicated that miR-671 promotes prostate cancer cell proliferation by targeting tumor suppressor sex-determining region Y-box 6 (22). A recent study indicated that miR-671-3p suppressed breast cancer progression (11). However, the function of miR-671-3p in NSCLC remains unclear. In the present study, the data demonstrated that miR-671-3p serves as a tumor suppressor. Using CCK-8 and Transwell assays, it was revealed that miR-671-3p inhibited NSCLC cell proliferation and invasion.

miRNAs have been demonstrated to target mRNAs for the regulation of gene expression in cancer (23). Through bioinformatics analysis, the present study identified that miR-671-3p may target CCND2. Using a luciferase reporter assay, the direct interaction between miR-671-3p and CCND2 mRNA was validated. Furthermore, it was demonstrated that miR-671-3p overexpression significantly inhibited the expression of CCND2 in NSCLC tissues. Previous studies imply that CCND2 is a classical oncogene in various types of cancer (24). CCND2 upregulation may lead to proliferation and metastasis of cancer cells: For example, Wang et al (25) indicated that miR-1297 represses the growth and metastasis of colorectal cancer by suppressing CCND2 expression.
CCND2 was also demonstrated to participate in NSCLC progression (24). Consistent with these previous studies, the present study also indicated that CCND2 exerts oncogenic roles in NSCLC. It was identified that the restoration of CCND2 expression may significantly attenuate the inhibitory effects of miR-671-3p mimics on NSCLC cell proliferation and invasion. Notably, according to the results of the target prediction, miR-671-3p may also target other genes. Whether other potential genes are involved in this process requires additional investigation.

In conclusion, the present study provided novel evidence to suggest the tumor suppressor role of miR-671-3p in NSCLC. Furthermore, CCND2 was identified as a novel target of miR-671-3p in NSCLC. Future studies will focus on whether miR-671-3p may serve as a biomarker and therapeutic target for NSCLC treatment.

\section{Acknowledgements}

Not applicable.

\section{Funding}

No funding was received. 


\section{Availability of data and materials}

All data generated or analyzed during this study are included in this published article.

\section{Authors' contributions}

YY and XF initiated and designed the study, and analyzed and interpreted the results. YZ performed RT-qPCR analysis. XF wrote the manuscript. All authors read and approved the final manuscript.

\section{Ethics approval and consent to participate}

For the use of human samples, the protocol for the present study was approved by the Institutional Ethics Committee of Ningbo No. 2 Hospital and all enrolled patients provided written informed consent.

\section{Patient consent for publication}

All patients within the present study provided consent for the publication of their data.

\section{Competing interests}

The authors declare that they have no competing interests.

\section{References}

1. Siegel R, Ma J, Zou Z and Jemal A: Cancer statistics, 2014. CA Cancer J Clin 64: 9-29, 2014

2. Ettinger DS: Ten years of progress in non-small cell lung cancer. J Natl Compr Canc Netw 10: 292-295, 2012.

3. Xiao F, Liu D, Guo Y, Shi B, Song Z, Tian Y, Zhang Z and Liang C: Survival rate and prognostic factors of surgically resected clinically synchronous multiple primary non-small cell lung cancer and further differentiation from intrapulmonary metastasis. J Thorac Dis 9: 990-1001, 2017.

4. Chen X, Du J, Jiang R and Li L: MicroRNA-214 inhibits the proliferation and invasion of lung carcinoma cells by targeting JAK1. Am J Transl Res 10: 1164-1171, 2018.

5. Saumet A and Lecellier CH: microRNAs and personalized medicine: Evaluating their potential as cancer biomarkers. Adv Exp Med Biol 888: 5-15, 2015.

6. Bartel DP: MicroRNAs: Target recognition and regulatory functions. Cell 136: 215-233, 2009.

7. Garzon R, Calin GA and Croce CM: MicroRNAs in cancer. Annu Rev Med 60: 167-179, 2009.

8. Ren P, Gong F, Zhang Y, Jiang J and Zhang H: MicroRNA-92a promotes growth, metastasis and chemoresistance in non-small cell lung cancer cells by targeting PTEN. Tumour Biol 37: 3215-3225, 2016.

9. Lv X, Li CY, Han P and Xu XY: MicroRNA-520a-3p inhibits cell growth and metastasis of non-small cell lung cancer through PI3K/AKT/mTOR signaling pathway. Eur Rev Med Pharmacol Sci 22: 2321-2327, 2018.
10. Li S, Zhang J, Zhao Y, Wang F, Chen Y and Fei X: miR-224 enhances invasion and metastasis by targeting HOXD10 in non-small cell lung cancer cells. Oncol Lett 15: 7069-7075, 2018.

11. Xiong DD, Chen H, He RQ, Lan AH, Zhong JC, Chen G, Feng ZB and Wei KL: MicroRNA-671-3p inhibits the development of breast cancer: A study based on in vitro experiments, in-house quantitative polymerase chain reaction and bioinformatics analysis. Int J Oncol 52: 1801-1814, 2018.

12. Livak KJ and Schmittgen TD: Analysis of relative gene expression data using real-time quantitative PCR and the 2(T)(-Delta Delta C) method. Methods 25: 402-408, 2001.

13. Jiang B, Li M, Ji F and Nie Y: MicroRNA-219 exerts a tumor suppressive role in glioma via targeting Sal-like protein 4. Exp Ther Med 14: 6213-6221, 2017.

14. Goldstraw P, Crowley J, Chansky K, Giroux DJ, Groome PA, Rami-Porta R, Postmus PE, Rusch V and Sobin L; International Association for the Study of Lung Cancer International Staging Committee; Participating Institutions: The IASLC lung cancer staging project: Proposals for the revision of the TNM stage groupings in the forthcoming (seventh) edition of the TNM classification of malignant tumours. J Thorac Oncol 2: 706-714, 2007.

15. Onat S, Ates G, Avci A, Yildiz T, Birak A, Akgul Ozmen C and Ulku R: The role of mediastinoscopy in the diagnosis of non-lung cancer diseases. Ther Clin Risk Manag 13: 939-943, 2017.

16. Yu YX, Wang Y and Liu H: Overexpression of PTEN suppresses non-small-cell lung carcinoma metastasis through inhibition of integrin $\alpha V \beta 6$ signaling. Am J Transl Res 9: 3304-3314, 2017.

17. Wang S, Zhang B, Li C, Cui C, Yue D, Shi B, Zhang Q, Zhang Z, Zhang $X$ and Wang C: Prognostic value of number of negative lymph node in patients with stage II and IIIa non-small cell lung cancer. Oncotarget 8: 79387-79396, 2017.

18. Esmatabadi MJD, Farhangi B, Montazeri M, Monfared H, Sistani RN and Sadeghizadeh M: Up-regulation of miR-21 decreases chemotherapeutic effect of dendrosomal curcumin in breast cancer cells. Iran J Basic Med Sci 20: 350-359, 2017.

19. Du L, Chen T, Zhao K and Yang D: miR-30a suppresses osteosarcoma proliferation and metastasis by downregulating MEF2D expression. Onco Targets Ther 11: 2195-2202, 2018.

20. Jeon SH, Yoo JK, Kim CM, Lim ES, Lee SJ, Lee JM, Oh SH and Kim JK: The novel hsa-miR-12528 regulates tumourigenesis and metastasis through hypo-phosphorylation of AKT cascade by targeting IGF-1R in human lung cancer. Cell Death Dis 9: $493,2018$.

21. Xu J, Pan X and Hu Z: MiR-502 mediates esophageal cancer cell TE1 proliferation by promoting AKT phosphorylation. Biochem Biophys Res Commun 501: 119-123, 2018.

22. Yu Y, Wang Z, Sun D, Zhou X, Wei X, Hou W, Ding Y, Ma Y and Hou Y: miR-671 promotes prostate cancer cell proliferation by targeting tumor suppressor SOX6. Eur J Pharmacol 823: 65-71, 2018.

23. Yang F, Wei K, Qin Z, Liu W, Shao C, Wang C, Ma L, Xie M, Shu Y and Shen H: MiR-598 suppresses invasion and migration by negative regulation of derlin-1 and epithelial-mesenchymal transition in non-small cell lung cancer. Cell Physiol Biochem 47: 245-256, 2018.

24. Li YL, Wang J, Zhang CY, Shen YQ, Wang HM, Ding L, Gu YC, Lou JT, Zhao XT, Ma ZL and Jin YX: MiR-146a-5p inhibits cell proliferation and cell cycle progression in NSCLC cell lines by targeting CCND1 and CCND2. Oncotarget 7: 59287-59298, 2016.

25. Wang Y, Xue J, Kuang H, Zhou X, Liao L and Yin F: microRNA-1297 inhibits the growth and metastasis of colorectal cancer by suppressing cyclin D2 expression. DNA Cell Biol 36: 991-999, 2017. 